\title{
Original $\mid$ THE USE OF ELECTRONIC PORTAL IMAGING DEVICE IN CORRECTION Article OF SETUP ERRORS DURING EXTERNAL BEAM RADIATION THERAPY REVIEW AND INITIAL CLINICAL EXPERIENCE SINGLE INSTITUTION STUDY. Mostafa El-Haddad ${ }^{1}$, Ahmad Habash ${ }^{2}$, Romany R. ${ }^{2}$
}

${ }^{1}$ Department of Clinical Oncology, Kasr EL-Ainy Oncology Centre (NEMROCK), ${ }^{2}$ Department of Clinical Physics, Kasr EL-Ainy Oncology Centre (NEMROCK), Cairo University, Egypt

\begin{abstract}
In this Review of current clinical practice of set-up error verification by means of portal imaging, we firstly define the various types of setup errors using a consistent nomenclature. The different causes of set-up errors are then summarized. Next, the efforts done to minimize patient set-up errors are presented in different body sites. Also we focus on the more recent studies in order to assess the criteria for good clinical practice in patient positioning. We then present the result of our preliminary experience in the use of electronic portal image device (EPID) for the first time in our department. This a retrospective analysis for the images taken to patients treated with three dimensional conformal radiation therapy (3D-CRT) in our department between October 2005 and July 2006. Our results in shift of set-up errors reported in different tumor sites in the $\mathrm{X}, \mathrm{Y}, \mathrm{Z}$ directions in addition to errors in rotation. The number of images in our studies was still low compared to the literatures, but still there were images from different body sites. Finally we are giving some recommendations, collected from many publications in the use of portal imaging devices in the correction of systematic and random set-up errors during routine clinical practice.
\end{abstract}

Key Words: Electronic portal image device, error correction

Corresponding Author: Mostafa El-Haddad, King Faisal Hospital,Riyad, Saudia ArabiaTel.:00966565354202

\section{INTRODUCTION}

Conformal radiotherapy aims at limiting toxicity of critical organs while maximizing the tumor dose. This goal can be partly achieved by minimizing the treatment field size. The treatment field size is mainly determined by the size of the tumor including subclinical disease and by the margins applied to compensate for geometric uncertainties that occur during radiotherapy. Following the nomenclature proposed by the International Commission on Radiation Units and Measurements (ICRU), the gross tumor volume plus subclinical disease is called clinical target volume (CTV), and the CTV plus safety margins is called the planning target volume (PTV) ${ }^{1}$. Minimization of these so-called CTV-to-PTV margins, will improve the conformity index and this will lead to a reduction of the irradiated volume and hence; normal tissue exposed to radiation. To decrease the irradiated volume, high precision radiation therapy is required. A fundamental tenet of radiation therapy is that successful outcomes require accurate alignment of the treatment beam to the target tissue ${ }^{2}$. Set-up errors can be measured using portal imaging by applying megavoltage film or an electronic portal imaging device (EPID). Megavoltage film measurements are rather time consuming and not always very accurate. Over the last years, EPIDs have become available in a large number of institutions to measure set-up errors ${ }^{3}$. Weekly portal filming is the routine clinical standard for ensuring accurate targeting of external beam radiation therapy ${ }^{4}$. The importance of geometric accuracy has driven the development of devices that have the potential to monitor treatment accuracy more effectively than weekly port filming, with minimal increase in work load ${ }^{4}$. Electronic portal imaging devices (EPIDs) can acquire images automatically with near real-time display, store them digitally, and provide quantitative analysis tools. Also electronic portal images (EPIs) can be acquired with higher frequency than film with only minimal additional costs. Studies have shown that increased portal imaging frequency can reveal daily variations in patient alignment that are not observed with weekly filming 5 . Furthermore, EPIDs provide the user with immediate patient alignment information, without the delay involved in processing a film. Instant image availability enables the development of on-line correction protocols and daily targeting adjustments ${ }^{6}$. In addition to aiding acquisition, the digital nature of electronic portal images (EPIs) can be exploited to enhance the portal review process. Studies have examined the process of subjective portal image evaluation by clinicians 
and have found a wide variation among reviewers in reporting setup deviations in port films ${ }^{7}$. The purpose of this paper is to review current practice on set-up error determination, summarize and discuss the results of our initial experience using the EPID. At the end we tried to make a clear recommendation for the use of portal imaging procedures to quantify and reduce set-up errors in clinical practice.

\section{Set-up Errors}

A patient set-up error is the difference between the actual and intended position of the part of the patient that is irradiated, with respect to the treatment beam(s) during treatment. The intended or reference patient position is recorded on a reference image, being either a simulator image or a digitally reconstructed radiograph (DRR). On the reference image, anatomical structures (e.g. bone, lung, and body contour), radio-opaque markers (if any) and the outline of the field which is used to generate the image are seen ${ }^{8}$. The patient treatment position is measured by making a megavoltage film or electronic portal image of the same field at the treatment unit ${ }^{9,10}$. The measured set-up error also includes errors introduced by the generation of the DRR or digitization of the simulator image and the portal image caused by a finite sampling resolution, which in principle can be known a priori from imaging theory. The inter-observer variation introduced by the manual matching of a reference image and a portal image can also introduce substantial measurement errors. $^{11}$

The ICRU supplement \#62 defined certain set-up errors factors to be considered when defining a planning target volume: The intrafractional variations (variations during single fraction) and the interfractional variations (variations during the entire course of treatment), each may be random e.g. physiological processes or systematic e.g. circulation (Table 1). This helped us to define the margin to be taken into the CTV to ensure that it receive the dose intentioned. When choosing the margins the risk of missing part of the CTV must be balanced against the risk of complications due to making the PTV too large.

\section{Efforts to minimize set-up errors}

As the course of therapy requires about 5 to 8 weeks to complete, setting up the patient exactly the same way every day in agreement with the treatment plan, which is based on the anatomy from the planning CT scan, is difficult, if not impossible. Even a few millimeters of displacement may result in substantial dose deviations. Therefore, precision in patient setup is of utmost importance in this new era of radiation therapy. Current noninvasive immobilization techniques used to prevent significant patient motion during the treatment cannot guarantee the accuracy and precision of daily setup ${ }^{12,13}$. Electronic portal imaging has been established as the gold standard for online verification of patient setup. If two or more portal images are acquired immediately before the dose delivery, the patient position can be verified and corrected. A number of correction strategies and workflows have been developed to facilitate the process and improve the alignment precision. Because of their inherent two dimensional (2D) natures, images from an EPID can only be compared with a small subset of the available 3D planning CT information. Digitally reconstructed radiographs (DRR) of reference points, such as implanted markers, are used as reference to be localized on the portal image. In sites such as the head and neck, where not much interfraction or intrafraction motion of the tumor relative to the bony anatomy occurs, bony landmarks are used for reference. However, identification of three anatomic features, visible from two perpendicular portal views, which is necessary for verification of out-of plane rotation, is difficult. This necessitate searching for a more precise methods for patient positioning and increased the interest in developing $3 \mathrm{D}$ imaging techniques that can verify the patient setup immediately before treatment. One such technique is cone-beam $\mathrm{CT},(\mathrm{CBCT})$ the reconstruction of $3 \mathrm{D}$ volumes from a series of $2 \mathrm{D}$ projection images, performed on the patient on the treatment table. The 3D reconstructed volume may be used to verify and correct the patient setup in the linear accelerator coordinate frame and compare the treatment patient position with the planning patient position as defined by the planning CT.

Table 1: Factors to be considered when defining a planning target volume.

\begin{tabular}{|c|c|c|c|c|}
\hline \multirow[b]{2}{*}{ Category } & \multicolumn{2}{|c|}{$\begin{array}{c}\text { Interfractional variations } \\
\text { (variations during single fraction) }\end{array}$} & \multicolumn{2}{|c|}{$\begin{array}{c}\text { Interfractional variations } \\
\text { (variations during the entire course of treatment) }\end{array}$} \\
\hline & Random & Systematic & Random & Systematic \\
\hline \multicolumn{5}{|l|}{ Variations of CTV } \\
\hline In size & $\begin{array}{l}\text { Physiological processes } \\
\text { (circulation, respiration, peristalsis) }\end{array}$ & $\begin{array}{l}\text { Physiological processes } \\
\text { (Circulation) }\end{array}$ & $\begin{array}{l}\text { Physiological processes(e.g.degree of } \\
\text { bladder filling, bowel gas) }\end{array}$ & Tumor reduction or swelling \\
\hline $\begin{array}{l}\text { In position relative } \\
\text { to a fixed point in the } \\
\text { patient }\end{array}$ & $\begin{array}{c}\text { Physiological processes } \\
\text { (circulation, respiration, peristalsis) }\end{array}$ & $\begin{array}{l}\text { Change in treatment position } \\
\text { (prone-supine) }\end{array}$ & $\begin{array}{l}\text { Physiological processes (e.g. degree of } \\
\text { filling, cavities) }\end{array}$ & Weight loss \\
\hline \multicolumn{5}{|c|}{ Variations in position of the patient } \\
\hline $\begin{array}{l}\text { relative to the } \\
\text { treatment beams }\end{array}$ & Patient movements & & Daily set-up & Technical errors \\
\hline
\end{tabular}


Although the concept of cone-beam CT was developed decades ago in the field of diagnostic and interventional radiology ${ }^{14,15}$, its application to radiation therapy is still in developmental stages. In radiation therapy, two approaches to CBCT are usually considered based on the type of beam used to form the image. For kilovoltage $(\mathrm{kV}) \mathrm{CBCT}$, a separate $(\mathrm{kV})$ source and a second dedicated EPID, attached perpendicular to the treatment beam, are used to acquire the projection images ${ }^{16,17}$. For megavoltage (MV) CBCT, the MV source of the linac and the EPID currently employed to measure portal images are used for imaging. Using the treatment beam for imaging is appealing because this application requires no additional hardware (along with the associated expense and maintenance), and the image is obtained in exact geometric coincidence with the treatment. Almost 20 years ago, researchers began using the linac beam for 3D imaging. Initially, research focused on reconstruction of $2 \mathrm{D}$ slices by use of fan-beam reconstruction ${ }^{18,19}$. Recently, MV fan-beam CT has been integrated into the helical tomotherapy system (Tomotherapy Inc, Madison, WI) ${ }^{20}$. As the technology of 2D X-ray detectors advances ${ }^{21}$, cone-beam reconstruction systems become increasingly feasible. Several researchers have demonstrated MV cone-beam CT by use of standard linacs and EPIDs ${ }^{22,23}$. MV CBCT has also been performed with utilization of the first generation of amorphous silicon (a-Si) portalimaging detectors ${ }^{24,25}$. In much of this early work, resolution was maximized by use of experimental equipment or application of high (50-200cGy) doses. Strategies to reduce doses to clinically acceptable values have included the development of more sensitive detectors ${ }^{26}$ or the restriction of the imaging volume to the treatment volume ${ }^{27,28}$. Seppi et al. ${ }^{26}$ recently reported high-contrast resolution while delivering only $16 \mathrm{cGy}$. There are a number of advantages of MVCT over planar megavoltage portal radiographs:

1. MVCTs are fully three-dimensional.

2. MVCTs have better soft-tissue contrast than planar radiographs.

3. MVCTs are easier to compare with planning CT images.

In addition to using the treatment beam to produce an MVCT scan, it is possible to acquire a kilovoltage CT scan at the time of treatment. Several approaches are being investigated. It is possible to put a conventional CT scanner in the treatment room ${ }^{29,30}$. The CT scanner may be driven on rails over the treatment couch. Alternatively, a couch may be designed to transport the patient either through the bore of the CT gantry for imaging or beneath the linear accelerator for treatment. The tissue contrast visible at a given resolution and given dose for a conventional $\mathrm{kVCT}$ is superior to that for an $\mathrm{MVCT}^{31}$. It is possible to put a kilovoltage X-ray tube and a detector array on board the linac to acquire CT scans. Because the gantry rotation of a linac is much slower than a $\mathrm{CT}$ ring gantry, flat-panel detectors are more practical from the perspective of throughput, so that a whole volume may be acquired with one or a few rotations..$^{32,33}$

\section{Other examples for other systems used to minimize set-up errors are:}

1. The optical-guidance systems ${ }^{34}$ which can greatly reduce the need for image guidance, but may not eliminate it completely ${ }^{35}$. Optical guidance is accomplished through detection of four passive markers that are attached to a custom bite plate that links to the maxillary dentition of the patient to form a rigid system. A camera system mounted to the ceiling of the linear accelerator vault and interfaced with a computer tracks the translations and rotation of the patient's head. Optically guided radiotherapy may enhance normal-tissue sparing, provide a high degree of conformality, and improve dose homogeneity characteristics in head-and-neck patients. ${ }^{36,37}$

2. Ultrasound images were among the first to be used to determine the patient's internal anatomy at the time of treatment ${ }^{38,39}$. A two-dimensional ultrasound beam can be scanned in the orthogonal dimension. The scanning ultrasound transducer can be tied to a mechanical arm that constrains the direction of the beam, and from this a partial 3D ultrasound image of the patient can be rendered ${ }^{40}$. Another approach is to optically track the position of the transducer, which eliminates the need for a mechanical attachment of the transducer ${ }^{39}$. The ultrasound image can be compared to the planning CT scan to determine if the patient's target volume is in the right place. Potentially, this system could be applied also to other abdominal sites, but its general utility will be limited by problems associated with limited penetration through many tissue types, including fat, bone, and lung. Also the accuracy of the ultrasound target volume delineation is usually operator dependent.

\section{PATIENTS AND METHODS}

This is a retrospective analysis for the images taken to patients treated with 3D-CRT in our department between October 2005 and July 2006.

Patient treatment process start on the CT simulator (Figure 1), where a CT scan in the treatment position using a suitable method for fixation, (e.g. Thermoplastic masks), vacuum air mattresses, was chosen for all patients, with 0.3 to $0.5 \mathrm{~cm}$ cut sections, pictures transferred through the DICOM (Digital Intercommunication in Medicine) network server to the planning system (PS) Xio [computer medical system, $(\mathrm{CMS} \circledast)]$ ].

After drawing the GTV, CTV and the PTV, our 


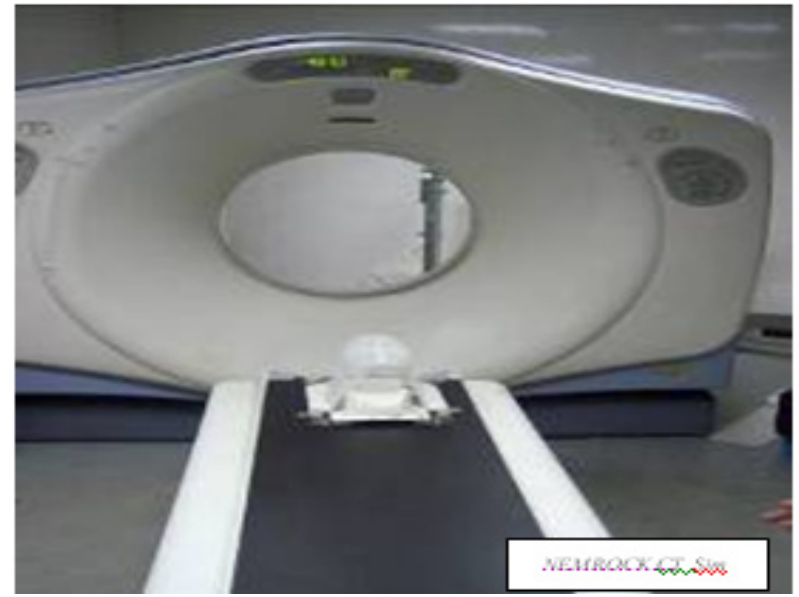

Fig. 1: CT simulator at NEMROCK.

physics team put different plans and the team (physician, physicists) set to accept and choose between different plans available. After the acceptance of a plan, the DRR images was captured digitally from the planning system and transferred and stored to the EPID computer system (iView GTTR Elekta) to serve as a reference image for EPID reviews. For patient positioning, marks placed on the patient skin and mask during simulation procedure was aligned with long lateral, sagittal, and transversal laser lines. Focus-to-skin distance was measured before each fraction. "Immobilization" devices are used both to assist in patient positioning and to reduce intra-treatment motion. For example, at our institution standard head supports and a custom-made thermoplastic masks are used to position and immobilize head and neck patients. Verification session with fields in two orthogonal directions acquired initially before starting treatment using ELEKTA EPID and matching between the DRR and the EPID picture was done to detect any obvious shift. Most of the correction set-up procedure follows the sequence of steps shown in figure 2. The patient is positioned, the therapists leave the room, and an EPI is acquired. The image is displayed on a computer and compared, visually, and digitally on the Iview ${ }^{\circledR}$ imager (Figure 3). In nearly every case, a physician decides whether the patient position is acceptable. Setup errors less than $5 \mathrm{~mm}$ generally are considered acceptable. If the setup is accepted, then the patient is treated. If the setup is rejected, then the therapists reenter the room to adjust the patient position. After the adjustment, image acquisition and registration must be repeated to verify that the patient position indeed has been corrected. Matching was done visually by the physician and the radiographer and also automatic match using the analysis tool of the EPID software, was done to detect the amount and the degree of the shift in the cranio-caudal, vertical and lateral directions along with rotation if any (Figure 3). Shift in Y direction was reported in our study once from the Anterior view or the lateral view not both. Rotations were reported in terms of degrees, whereas translational deviations were given as centimeters at isocenter. If the angle of treatment field does not allow image capture (e.g. table collision with the EPID) such in cases with non coplanar fields, separate fields with reasonable size to show the anatomy in two orthogonal directions were used, only for treatment set-up and not for treatment. Before the end of treatment course our physics team calculate the total Monitor units (MUs) used for these portal images and decrease it (if needed) from the whole treatment dose, however this was never of clinical significance. Portal images used for this work were single or double exposure images that indicate the boundaries of the treatment portal and show additional anatomy beyond the treatment aperture. The MUs used was between 6 and 8 using $6 \mathrm{MV}$ energy. Out of 160 successive images only 103 images used for treatment set-up correction were analyzed, 57 images were excluded either because of very bad quality or there was no a clear bony landmarks that allow easy comparison. With anterior views we were able to measure set-up error in the $\mathrm{X}$ and $\mathrm{Y}$ directions, while the lateral view give us the errors in the $\mathrm{Z}$ and again the $\mathrm{Y}$ directions.

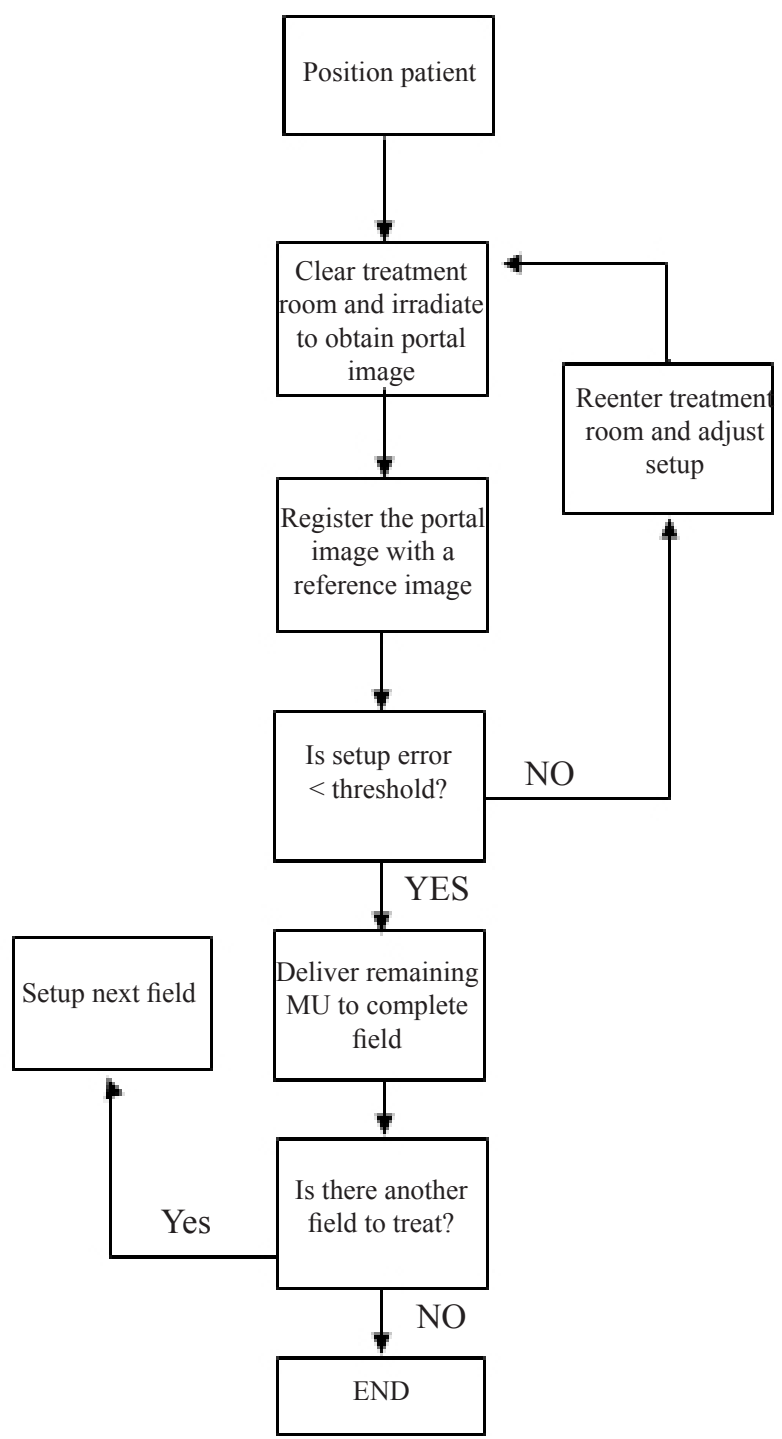

Fig. 2: Common treatment sequence used for the on-line correction of setup errors detected with an EPI device. 


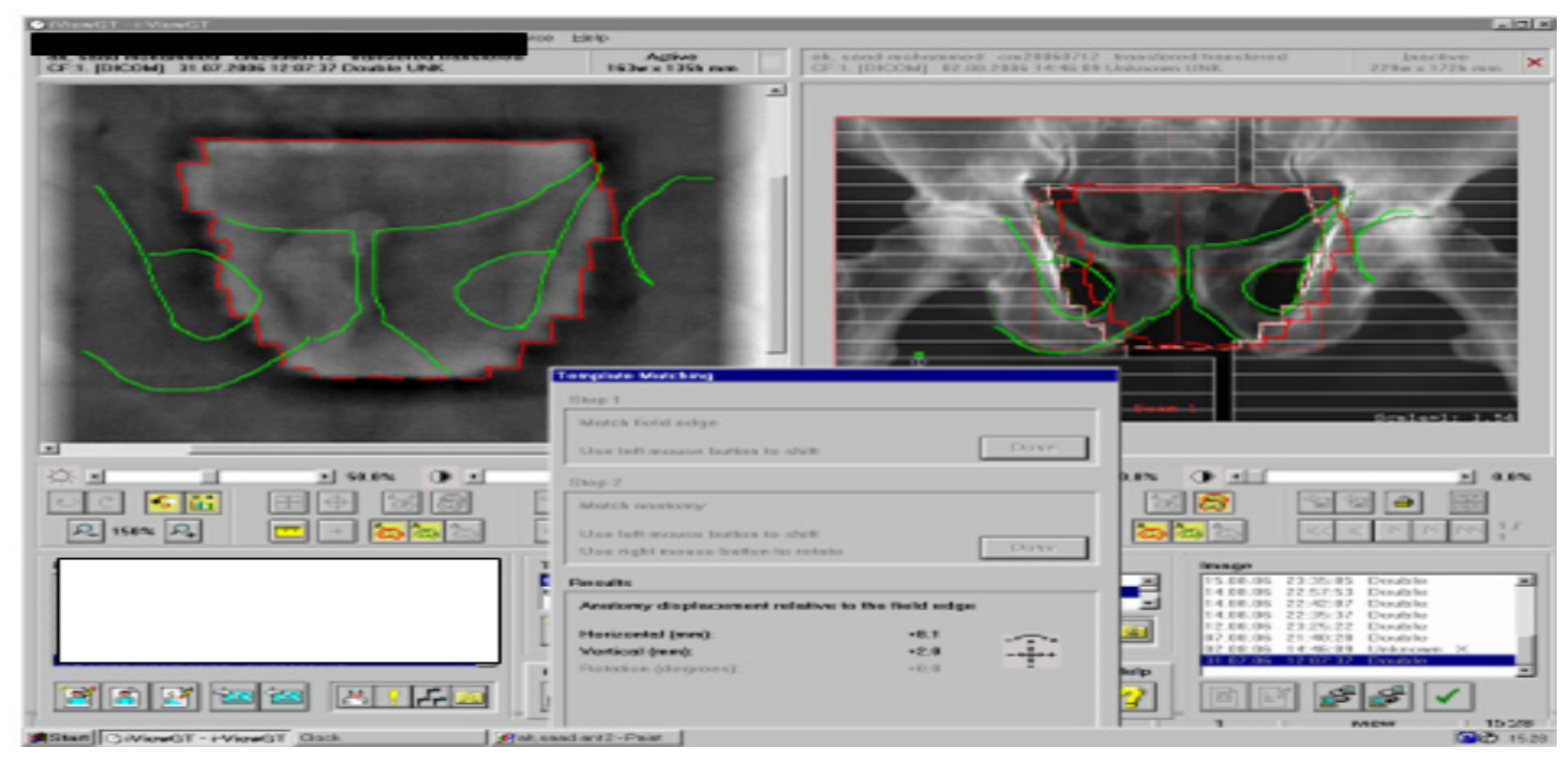

Fig. 3: EPIs for a patient with prostate cancer compared to the DRR visually and digitally.

\section{RESULTS}

\section{Treatment sites}

The EPIs was taken for patients under treatment with 3D CRT for different sites in the body, 19 images for brain tumors, 36 for prostate cancer, five for bronchogenic cancer, 17 for head and neck, 21 breast (Rt. and Lt.), and five cases for Abdominal malignancies, a total of 103 views.

Detailed shifts in the $\mathrm{X}, \mathrm{Y}$, and $\mathrm{Z}$ direction along with rotation degrees presented in (Table 2). The mean shift in the horizontal ( $\mathrm{X}$ direction) direction was 2.4 $\mathrm{mm}$ and a median of $1.8 \mathrm{~mm}$ with Standard deviation of \pm 2.6 . maximum of $13 \mathrm{~mm}$ and a minimum of 0 . The mean shift in longitudinal direction (Y direction), was 2.9 $\mathrm{mm}$ and a median of $1.2 \mathrm{~mm}$ with Standard deviation of \pm 2.3 maximum of $14 \mathrm{~mm}$ and a minimum of 0 . The mean shift in the vertical ( $Z$ direction) direction was $0.7 \mathrm{~mm}$ and a median of $0 \mathrm{~mm}$ with Standard deviation of \pm 1.8 . maximum of $9.9 \mathrm{~mm}$ and a minimum of 0 . Rotation angle ranged from 0 degree to 10 degree (Table 3 ).

In Brain region images showed a median shift of $2 \mathrm{~mm}$ $(\mathrm{SD} \pm 4.8)$ and $1.9 \mathrm{~mm}( \pm 3.3)$ in the $\mathrm{Y}$ and $1.6 \mathrm{~mm}( \pm 2.25)$ in the $\mathrm{Z}$ direction. For the head and neck images there was a median shift of $1.2 \mathrm{~mm}( \pm 0.8), 1.15 \mathrm{~mm}( \pm 1.6)$ and $0.7( \pm 3.5)$ in the $\mathrm{X}, \mathrm{Y}, \mathrm{Z}$ directions, respectively, almost there was no reported rotation. In prostate images the shift was $1.5 \mathrm{~mm}( \pm 1.7) 2.9 \mathrm{~mm}( \pm 3.2)$, and $2.2( \pm 2.32) \mathrm{mm}$ in the $\mathrm{X}, \mathrm{Y} \mathrm{Z}$ directions, respectively. In the abdomen region median shift was $3 \mathrm{~mm}$ in the $X$ direction and $1.5 \mathrm{~mm}$ in the $\mathrm{Y}$ and 0 in the $\mathrm{Z}$ direction Standard deviation was $\pm 1.3, \pm 4.1$ and \pm 0.9 , respectively. The last few patients in chest region shift were reported in the $\mathrm{X}$ and $\mathrm{Y}$ direction only as all four patients treated by Anterior-posterior (A/ $\mathrm{P})$, and Posterior anterior (P/A) fields.

Table 2: Median, Mean, SD, Minimum, Maximum and Mode, in the X, Y and Z direction along with rotation degrees.

\begin{tabular}{lcccc}
\hline & Shift in the $\mathbf{X}$ direction & Shift in the $\mathbf{Y}$ direction & Shift in the $\mathbf{Z}$ direction & Shift in Rotation \\
\hline Median & 1.8 & 1.7 & 1.2 & 0 \\
Mean & 2.44 & 2.9 & 2.1 & 0.8 \\
SD & 2.63 & 3 & 2.3 & 1.86 \\
Minimum & 0 & 0 & 0 & 0 \\
Maximum & 13.7 & 14 & 12.1 & 9.9 \\
Mode & 2.3 & 0 & 0.5 & 0 \\
\hline
\end{tabular}

SD: Standard deviation 
Table 3: Detailed shifts in the $\mathrm{X}, \mathrm{Y}$ and $\mathrm{Z}$ direction along with rotation degrees.

\begin{tabular}{|c|c|c|c|c|c|c|}
\hline \multirow[t]{2}{*}{ Filed number } & \multirow[t]{2}{*}{ Field Orientation } & \multirow[t]{2}{*}{ Treatment Site } & \multicolumn{4}{|c|}{ Shift in $\mathrm{mm}$} \\
\hline & & & $\mathrm{X}$ direction & $Y$ direction & $\mathrm{Z}$ direction & Rotation \\
\hline 1 & Anterior & Brain & -3 & 0 & - & 0 \\
\hline 2 & Anterior & Brain & 0.2 & 1.2 & - & 0 \\
\hline 3 & Anterior & Brain & -0.4 & -0.8 & - & 0 \\
\hline 4 & Anterior & Brain & -2 & -3.3 & - & 0 \\
\hline 5 & Anterior & Brain & -2.6 & -4.4 & - & 0 \\
\hline 6 & Anterior & Brain & -0.3 & 4 & - & 0 \\
\hline 7 & Anterior & Brain & 13.7 & 9.7 & - & 0 \\
\hline 8 & lateral & Brain & - & -4 & 2 & 0 \\
\hline 9 & lateral & Brain & - & 1 & 1 & 0 \\
\hline 10 & lateral & Brain & - & 0.4 & 0.5 & 0 \\
\hline 11 & lateral & Brain & - & 1.9 & 1.9 & 0 \\
\hline 12 & lateral & Brain & - & -12 & 1.1 & 0 \\
\hline 13 & lateral & Brain & - & -0.2 & 6.2 & 0 \\
\hline 14 & lateral & Brain & - & 0.5 & 1.3 & 0 \\
\hline 15 & lateral & Brain & - & 0.5 & 0.7 & 0 \\
\hline 16 & lateral & Brain & - & 7 & 1.2 & 0 \\
\hline 17 & lateral & Brain & - & -4.2 & -3 & 0 \\
\hline 18 & lateral & Brain & - & 1.5 & -2.7 & 0 \\
\hline 19 & lateral & Brain & - & -4 & 7.7 & 0 \\
\hline 20 & lateral & Prostate & - & -0.7 & 1.5 & 0 \\
\hline 21 & lateral & Prostate & - & 4.1 & 5.6 & -1.5 \\
\hline 22 & lateral & Prostate & - & 0 & -0.4 & 0 \\
\hline 23 & Ant. Oblique & Prostate & - & -0.1 & -0.2 & 0 \\
\hline 24 & Ant. Oblique & Prostate & 0.7 & -0.4 & 06 & 0 \\
\hline 25 & Ant. Oblique & Prostate & -4.8 & 0 & 0.2 & 0 \\
\hline 26 & Ant. Oblique & Prostate & -0.7 & 0.2 & -0.2 & 0 \\
\hline 27 & Post. Oblique & Prostate & 4.1 & -4.4 & -3.7 & 3.6 \\
\hline 28 & Post. Oblique & Prostate & 0 & 1.6 & -0.9 & 0 \\
\hline 29 & Anterior & Prostate & -0.1 & 1.7 & - & 0 \\
\hline 30 & Anterior & Prostate & -1.2 & -3.8 & - & 0 \\
\hline 31 & Anterior & Prostate & -0.9 & -3.6 & - & 0 \\
\hline 32 & Anterior & Prostate & -0.3 & 6.3 & 3.7 & 0 \\
\hline 33 & Ant. oblique & Prostate & 07 & 1.7 & 0 & 0 \\
\hline 34 & Post. Oblique & Prostate & 2.3 & -3.8 & -6.4 & 0 \\
\hline 35 & Post. Oblique & Prostate & -4.6 & 0.5 & 5.3 & 0 \\
\hline 36 & lateral & Prostate & - & -4.7 & 5 & 0 \\
\hline 37 & lateral & Prostate & - & -3.8 & 3.2 & 0 \\
\hline 38 & lateral & Prostate & - & -8.3 & -7 & 0 \\
\hline 39 & Anterior & Prostate & 2.3 & -5.3 & - & 0 \\
\hline 40 & Anterior & Prostate & -4.6 & -14 & - & 0 \\
\hline 41 & lateral & Prostate & - & 3.5 & 0.5 & 0 \\
\hline 42 & lateral & Prostate & - & -12.4 & -1.5 & 0 \\
\hline 43 & lateral & Prostate & - & -1 & 4.7 & 0 \\
\hline 44 & lateral & Prostate & - & -5.7 & -6 & 0 \\
\hline 45 & lateral & Prostate & - & -6 & -1.2 & 0 \\
\hline 46 & lateral & Prostate & - & -6 & 2.3 & 0 \\
\hline 47 & lateral & Prostate & - & 0.7 & - & 0 \\
\hline 48 & Anterior & Prostate & 5.6 & 2.3 & - & 0 \\
\hline 49 & Anterior & Prostate & 2.3 & -0.7 & - & 0 \\
\hline 50 & posterior & Prostate & -1 & 2 & - & 0 \\
\hline 51 & posterior & Prostate & 2.3 & 1.3 & 0.7 & - \\
\hline 52 & lateral & Prostate & - & -3.6 & 6 & - \\
\hline
\end{tabular}




\begin{tabular}{|c|c|c|c|c|c|c|}
\hline 53 & lateral & Prostate & - & -0.7 & -0.9 & - \\
\hline 54 & lateral & Prostate & - & -1.2 & 2.2 & - \\
\hline 55 & lateral & Prostate & - & -5 & -4.6 & - \\
\hline 56 & lateral & Prostate & - & 0 & 0 & - \\
\hline 57 & lateral & Prostate & - & 0 & -1 & 0 \\
\hline 58 & lateral & Head and neck & - & 0.5 & 0.6 & -1.8 \\
\hline 59 & lateral & Head and neck & - & 0 & 0.7 & 0 \\
\hline 60 & lateral & Head and neck & - & 2.9 & 0.6 & 0 \\
\hline 61 & lateral & Head and neck & - & 1.9 & -2.9 & 0 \\
\hline 62 & lateral & Head and neck & - & 1.4 & 2.4 & 0 \\
\hline 63 & lateral & Head and neck & - & -1.1 & -0.5 & 0 \\
\hline 64 & lateral & Head and neck & - & -1.2 & -0.8 & 0 \\
\hline 65 & lateral & Head and neck & - & 1.4 & 0 & 0 \\
\hline 66 & lateral & Head and neck & - & -6.1 & -12.1 & 3.1 \\
\hline 67 & lateral & Head and neck & - & -0.2 & - & 9.9 \\
\hline 68 & Anterior & Head and neck & 0.2 & -0.6 & - & 0 \\
\hline 69 & Anterior & Head and neck & -1.2 & 1.3 & - & 0 \\
\hline 70 & Anterior & Head and neck & 1.3 & 4 & - & 0 \\
\hline 71 & Anterior & Head and neck & 1.1 & 0.6 & - & 1.8 \\
\hline 72 & Anterior & Head and neck & -2.3 & -4.9 & 0.6 & -0.3 \\
\hline 73 & Med. Tang & breast & - & -9 & -0.3 & 0 \\
\hline 74 & Lat. Tang & breast & - & -4.2 & 4.7 & 0 \\
\hline 75 & Med. Tang & breast & - & -1.3 & -2.9 & 0 \\
\hline 76 & Lat. Tang & breast & - & 2.9 & 2.7 & 0 \\
\hline 77 & Med. Tang & breast & - & -10.7 & 5.7 & -1.5 \\
\hline 78 & Lat. Tang & breast & - & -6.3 & -2 & -7.1 \\
\hline 79 & Med. Tang & breast & - & -2.8 & 0.4 & -2.7 \\
\hline 80 & Lat. Tang & breast & - & -9.5 & 3.4 & -5.7 \\
\hline 81 & Med. Tang & breast & - & 1.3 & -0.4 & -5.2 \\
\hline 82 & Lat. Tang & breast & - & 2.4 & 2.3 & -1.2 \\
\hline 83 & Med. Tang & breast & - & -0.2 & 0 & -3.4 \\
\hline 84 & Lat. Tang & breast & - & -2 & -0.3 & -6.3 \\
\hline 85 & Med. Tang & breast & - & -4.8 & 1.9 & 2.6 \\
\hline 86 & Lat. Tang & breast & - & 2.2 & -1.9 & 4.4 \\
\hline 87 & Med. Tang & breast & - & -2.2 & 0.5 & 6.7 \\
\hline 88 & Lat. Tang & breast & - & 0.7 & 0.9 & 3.4 \\
\hline 89 & Med. Tang & breast & - & 1.1 & 0.7 & -0.7 \\
\hline 90 & Lat. Tang & breast & - & 1.6 & -0.6 & -0.4 \\
\hline 91 & Med. Tang & breast & - & 0 & 0.5 & 0 \\
\hline 92 & Lat. Tang & breast & - & 0.1 & 0 & 0 \\
\hline 93 & Med. Tang & breast & - & 0.1 & -0.5 & 0 \\
\hline 94 & Anterior & Abdomen & -2.8 & -7.9 & -0.5 & 0 \\
\hline 95 & Anterior & Abdomen & 3.5 & -8.1 & - & 0 \\
\hline 96 & Anterior & Abdomen & 3.2 & 1.5 & -2.1 & 0 \\
\hline 97 & posterior & Abdomen & - & 1.5 & - & 0 \\
\hline 98 & posterior & Abdomen & -0.5 & 2.3 & - & 0 \\
\hline 99 & Anterior & Chest & -1.5 & -0.4 & - & 0 \\
\hline 100 & Posterior & Chest & -1.6 & 0 & - & 0 \\
\hline 101 & Anterior & Chest & 2 & 0.6 & - & 0 \\
\hline 102 & Anterior & Chest & 9.1 & & - & 0 \\
\hline 103 & Anterior & Chest & -5 & & - & 0 \\
\hline
\end{tabular}

Ant.: Anterior, Post: Posterior, the minus (-) sign in the $\mathrm{X}$ direction means shift is to the rt. of the screen (Rt. side of the patient if the patient is prone and to the 1 t. side of the patient if the patient is lying prone, in the $\mathrm{Y}$ direction means caudally, in the $\mathrm{Z}$ direction means anterior of the patient, in the angle of rotation means to the Rt. 


\section{DISCUSSION}

For patients treated in the conventional manner, a setup error of 5 to $10 \mathrm{~mm}$ may be acceptable as the tumor still receive the dose prescribed (i.e., with large fields having wide margins), improved accuracy is desired for three-dimensional conformal radiotherapy (3-DCRT) and intensity-modulated treatments. With improved accuracy, treatment planning margins can be reduced; and the dose to nearby critical structures thereby decreased. In some cases, this reduction in normal tissue dose permits one to deliver a larger tumor dose and increase the probability of local tumor control without a concomitant increase in normal tissue complication rates.

A successful outcome, however, requires that every effort be made to position the target accurately at each treatment session.

Our study is a retrospective analysis of images taken during routine patient treatment with $3 \mathrm{D}-\mathrm{CRT}$ technique, although this resulted in low number of images and inadequate distribution of images types, however this also eliminated the extra care that we may give to treatment setup when we are running a clinical study.

\section{Set-up errors for head and neck and Brain treatments}

Most of EPIDs studies ${ }^{41-44}$, showed that the standard deviations of the systematic error was around 1.6-4.6mm and are generally larger than the standard deviations of the random error 1.1-2.5 $\mathrm{mm}$. With the currently available equipment, a standard deviation of $2 \mathrm{~mm}$ or less for both the random and systematic setup error can be considered 'state of the art'; i.e. good clinical practice. In our study we didn't compare systematic error versus random error, we reported only random errors, which were in general acceptable, the number of views in head and neck was very small and most of the images was lateral views with only five anterior view which do not give us an idea about any shift in the lateral $(\mathrm{X})$ direction. In our brain images there was a large shift $(13 \mathrm{~mm})$ in one patient which was due misplacement of the isocentre during simulation process and this was detected and corrected before start of treatment.

\section{Set-up errors for pelvic and Abdomen treatments}

The predominance of set-up errors in the anteriorposterior direction found in some studies ${ }^{41,43}$, is mainly due to the use of skin marks to determine the isocentre height in combination with the use of the pelvic bones as a match structure. The movement of the skin marks used for patient positioning relative to the pelvic bones, results in a set-up error. The skin movement might be due to respiration, weight loss or relaxation of the patient. This movement is expected to be small in the cranial-caudal and medial-lateral direction and more pronounced in the anterior-posterior direction.

There is no clear predominance of set-up errors in one direction when all pelvic studies are taken together ${ }^{45}$. In our study the median shift for prostate cancer patients (pelvis) was $1.5 \mathrm{~mm}$ (range: $0-5.6$ ), in the $\mathrm{X}$ direction, $2.9 \mathrm{~mm}$ (range: 0 - 14) in the Y direction and $2.2 \mathrm{~mm}$ (range: $0-7)$ in the $Z$ direction however our patients number still low and we didn't include other pelvic tumors which may need whole pelvic irradiation, also female patients tends to be more obese hence the set-up may be more difficult. We are conducting a prospective trial assessing set-up errors in pelvic tumors. There is little detailed information about set-up errors comparing prone versus supine patient position ${ }^{46}$. Therefore, no recommendations concerning this topic can be given based on set-up error studies. There has been much discussion about the effect of immobilization on positioning reproducibility.

For example, Bieri et al. concluded that immobilization did not reduce set-up errors ${ }^{47}$. In a letter to the editor, Van den Heuvel and Verellen ${ }^{48}$ question the conclusions drawn from this study, arguing that various reasons such as a too small patient sample, large inter-observer variations and unclear parameters to quantify set-up errors may have attributed to the absence of a significant correlation. There are a number of reasons why the Bentel et al. ${ }^{49}$ and Soffen et al..$^{50}$ in particular, and other studies concerning the impact of immobilization on setup errors, produce contradictory results. Firstly, studies based on data from different institutions are biased by differences in set-up reproducibility caused by other factors than immobilization ${ }^{51}$. Secondly, the specific custom-made details of the immobilization device and its precise use are important factors influencing the attainable reproducibility. The dimensions of the cast determine which part of the patient is fixed, for example including or excluding the knees. The experience and skill of the technicians constructing the casts influence the fit and therefore its immobilization characteristics. The marking of the isocentre on the skin, which moves relative to the cast from fraction to fraction, will result in a different set-up reproducibility compared to marking the isocentre on the cast. The mark on the cast will also not wear off with time, as occurs with the marks on the patient's skin. Thirdly, a large observer variance decreases the statistical significance of any difference in set-up error related to the use of different immobilization devices ${ }^{47}$. Fourthly, prospective studies may have been carried out more careful than retrospective studies, leading to better results. Comparison of prospective with retrospective studies may therefore lead to wrong conclusions. Fifthly, the time period at which portal images are acquired during a course of radiation therapy varies widely. Some studies analyzed portal images taken on one of the first days of treatment, others used portal images taken over the entire course of treatment, thereby 
incorporating possible time trends. In order to determine the influence of immobilization on set-up reproducibility, one has to compare the set-up accuracy of patients treated with and without immobilization, within one institution, treated during the same period of time, preferably in a randomized study. Furthermore, the set-up accuracy with and without immobilization should be evaluated similarly. Although the use of immobilization devices for the reduction of rotations around the right-left axis is controversial, it seems appropriate to try to immobilize the legs by means of, for instance, a knee-support. ${ }^{52}$

If marking of the isocentre is performed on a cast, the sides of the cast have to be high enough ${ }^{45}$. When skin marks are used, the sides of the cast have to be kept low, preventing overstretching of the skin when patients get into the mould..$^{53}$

In general, a standard deviation of $2.5 \mathrm{~mm}$ and $3.0 \mathrm{~mm}$ or less for the random and systematic set-up error can be considered 'state of the art' for prostate and pelvic treatment techniques, respectively. This accuracy can be reached with or without immobilization (and without the use of set-up corrections).

In our department we initially used the pelvi-cast (Med Tec $\AA$ ) in our prostate patients then we faced a problem in obese patients that skin marks shift during fixation of the pelvi-cast, and we found that putting the patient in supine position may be more reproducible and much easier for the radiotherapist for set-up. We recently started to use the foot-knee support (Figure 4) and we put extra laser marks on the patient legs for repositioning, and so far we didn't face any set-up problems. In obese patient we found that prone position is not practical and the loose abdomen prevent reproducibility.

\section{Set-up errors for lung treatments}

Because the structures near the lung move due to

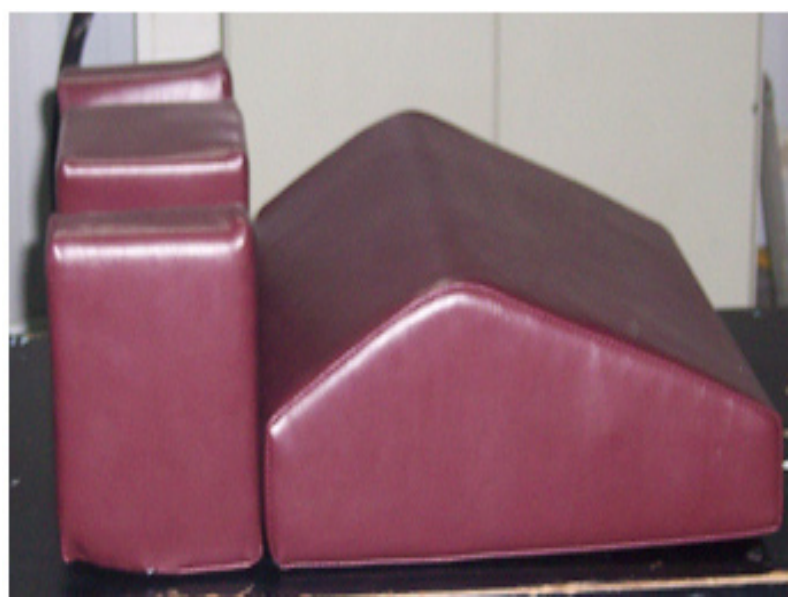

Fig. 4: Foot Knee support used in prostate cancer patients. breathing, a proper choice of the match structure to quantify set-up errors is important ${ }^{54}$. If only the vertebrae are used, breathing motion does not contribute much to the set-up error in the anterior-posterior direction, which would be the case when the ribs and the sternum are used. Furthermore, organ motion of, for example, the anterior part of the lung can be large relative to the vertebrae, while at the same time it is comparatively small relative to the sternum. Thus, the measured set-up error and the measured organ motion both depend on the choice of the match structure. If organ motion and setup errors are to be taken into account in order to apply appropriate field margins, the same match structure should be used to quantify both organ motion and set-up errors. The method of patient anatomy data collection, for example using breathing controlled CT-scanning ${ }^{55}$, is therefore as important as the determination of set-up errors. Intra-fraction errors which are more pronounced for the thoracic region than for other treatment regions can possibly be reduced using respiration gated therapy ${ }^{56}$. The limited amount of data currently available indicates that the size of the standard deviation of the systematic and random errors is about equal and standard deviations of systematic and random errors of $3.5 \mathrm{~mm}$ or less can be considered as 'state of the art'. The set-up errors for lung cancer irradiations are generally larger than set-up errors for prostate and head and neck treatments. This is mainly caused by the lack of rigid structures like the skull or hip bones, which can be used to immobilize the patient, and breathing motion. In our study only five images was found because by that time we didn't include many patients with bronchogenic carcinoma, we used for set-up the wing board which we constructed in house (Figure 5).

\section{Set-up errors for breast treatments}

Most studies use the central lung distance (CLD) and the cranial-caudal displacement to quantity set-up errors for breast treatment. The CLD is a good estimate for the

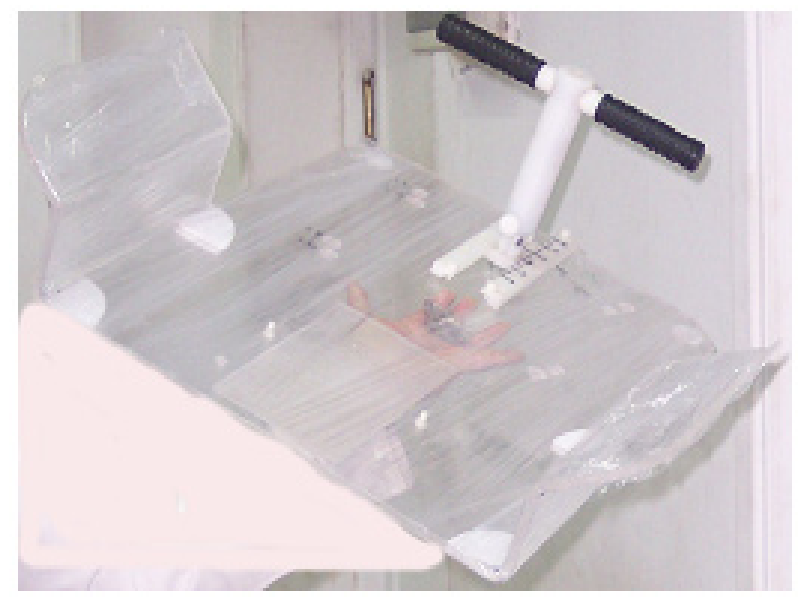

Fig. 5: Wing board used a method for patients positioning reproducibility. 
amount of lung tissue irradiated ${ }^{5,57}$, and the cranial-caudal displacement is important to determine the cranialcaudal set-up margins and the accuracy of the cranial match plane when also the supraclavicular and axillary nodes are treated, ${ }^{58,59}$. In general, the resulting deviations were larger than deviations observed for lung or pelvic treatments. However, the errors were judged clinically acceptable by the clinicians involved in these studies. The size of the standard deviation of the systematic errors is not well correlated with the standard deviation of the random error. In some studies the random error dominates, while in other studies the opposite is found. The use of a fixation device other than an arm rest has not been proven to improve patient stability. However, it is argued that when the use of a lung shielding block or collimator rotation is prevented, (i.e. by applying a wedge board), a considerable source of errors other than set-up errors (e.g. block misplacement) is excluded ${ }^{60}$. In our study we found very few pictures for Supraclavicular field and were of very bad quality that we couldn't assess properly, and we are stressing on it in our on going study. Shift in cranio-caudal direction was more pronounced than in the Anterio-posterior direction and required correction.

\section{Limitation of Epids}

EPIDs and EPIs do not give us any information about the tumor itself, whether or not it moves during treatment, also information about its size is it the same, increased or even decreased. Also still it does not tell us whether or not the patient moved during treatment session, so it's not suitable for on-line set-up correction.

EPIDS verification increase the time on the machine for each patient however it still faster and can be taken more frequently than the portal films with no additional cost, on the long run.

De Neve et al. reported that the mean increase in treatment time on the machine was $46 \%$, with a range of 7.7 to $442 \%{ }^{61}$. A 15 -minute treatment time therefore was increased to 21.9 minutes, on average, with a range of 16.2 minutes to over an hour.

For most institutions this fairly large increase in treatment time, combined with the need to have a physician available to review each setup.

\section{Sources of errors}

A number of sources of random and systematic population-based set-up errors can be distinguished. These mainly concern mechanical shortcomings (e.g. laser misalignment), or are patient related (e.g. skin mark movement), or fixation related (e.g. patient mobility). One other major factor influencing the set-up uncertainty is the accuracy with which the radiation technologists are able to position the patient using the set-up marks. This ability is influenced by the experience, previous training and concentration of the radiation technologist, as well as the time available to position the patient. The physical and mental state of the patient also influences the set-up accuracy.

\section{RECOMMENDATIONS}

Based on this study, the following procedures to quantify, report and reduce patient set-up errors are recommended:

1. Perform portal imaging during a few treatment sessions over a course of treatment for a limited number of patients for all treatment techniques of which the setup accuracy is unknown and of importance for the success of the treatment. Such a procedure might reveal unexpectedly large errors and establishes the general accuracy of a technique.

2. Analyze and report the measurement data using the distinction between the two main error classes: random and systematic errors. The errors should be analyzed independently along the orthogonal axes, and should preferably not only be given in terms of vector lengths. If random and systematic set-up errors are found which are much larger than the 'state of the art' limits given in this paper, every effort should be done to reduce these errors.

3. Systematic errors should be traced and reduced. If adjustments in the set-up procedure have been made, it is important to verify again by portal imaging, preferably by performing a randomized study, if the change in the procedure has indeed led to an improved patient set-up.

4. Perform portal imaging once or twice during the first week of treatment, for irradiation techniques which do not require a high accuracy of patient positioning, (e.g. some palliative treatments and mantle field irradiations).

5. Portal imaging should be performed for all patients to detect large deviations, which could affect the outcome of the treatment ${ }^{35,46}$. A simple set-up correction protocol can be applied on a routine base by radiation technologists, for example, visual inspection of the image with correction of errors larger than $1 \mathrm{~cm}$. If clinically important set-up errors are easily detectable using the light field, (e.g. for some tangential breast irradiation techniques of which the fields are not matched to parasternal fields or axillary fields), portal imaging may not be necessary on a routine bases.

6. Perform portal imaging several times at the start of 
a course of treatment and afterwards on a regular interval (weekly), for treatments which require a high accuracy, e.g. conformal techniques. This procedure should be combined with the application of a patient positioning correction protocol if one wants to attain set-up errors smaller than the current 'state of the art' that can be reached without correction protocols.

7. To use automated matching of a reference image with portal images if a small measurement error is required, choose a rigid and stable match structure, for example, pelvic bones in pelvic treatments, excluding the femoral shafts and two or more structures in the determination of set-up errors in lung cancer treatments. If needed, make large, lowdose, localization images to properly identify chosen match structures.

8. Use high quality fixation devices. In general, the effect of immobilization on set-up errors depends strongly on the implementation and its clinical use, and is therefore institution dependent. One should compare the set-up accuracy of patients treated with two different types of immobilization, treated during the same period of time, preferably in a randomized study.

9. To provide proper training of radiation technologists in order to improve their skills to position a patient according to the treatment plan.

\section{REFERENCES}

1. International commission on radiation units and measurements. ICRU report 50: Prescribing, recording, and reporting photon beam therapy. 1993 .

2. Marks JE, Haus AG, Sutton HG, Griem ML. The value of frequent treatment verification films in reducing localization error in the irradiation of complex fields. Cancer 1976 Jun;37(6):2755-61.

3. Killoran JH, Kooy HM, Gladstone DJ, Welte FJ, Beard CJ. A numerical simulation of organ motion and daily setup uncertainties: Implications for radiation therapy. Int J Radiat Oncol Biol Phys 1997 Jan 1;37(1):213-21.

4. Meertens H, van Herk M, Bijhold J, Bartelink H. First clinical experience with a newly developed electronic portal imaging device. Int J Radiat Oncol Biol Phys 1990 May;18(5):1173-81.

5. Dong L, Boyer AL. An image correlation procedure for digitally reconstructed radiographs and electronic portal images. Int $\mathrm{J}$ Radiat Oncol Biol Phys 1995 Dec 1;33(5):1053-60.

6. Boyer AL, Antonuk L, Fenster A, Van Herk M, Meertens H, Munro P, et al. A review of electronic portal imaging devices (EPIDs). Med Phys 1992 Jan-Feb;19(1):1-16.
7. Bissett R, Boyko S, Leszczynski K, Cosby S, Dunscombe P, Lightfoot N. Radiotherapy portal verification: An observer study. Br J Radiol 1995 Feb;68(806):165-74.

8. Yin FF, Rubin P, Schell MC, Wynn R, Raubertas RF, Uschold G, et al. An observer study for direct comparison of clinical efficacy of electronic to film portal images. Int J Radiat Oncol Biol Phys 1996 Jul 15;35(5):985-91.

9. Bissett R, Leszczynski K, Loose S, Boyko S, Dunscombe P. Quantitative vs. Subjective portal verification using digital portal images. Int J Radiat Oncol Biol Phys 1996 Jan 15;34(2):489-95.

10. Michalski JM, Graham MV, Bosch WR, Wong J, Gerber RL, Cheng A, et al. Prospective clinical evaluation of an electronic portal imaging device. Int J Radiat Oncol Biol Phys 1996 Mar 1;34(4):943-51.

11. Gilhuijs KG, El Gayed AA, Van Herk M, VijlbriefRE. An algorithm for automatic analysis of portal images: Clinical evaluation for prostate treatments. Radiother Oncol 1993 Nov;29(2):261-8.

12. Xing L, Lin Z, Donaldson SS, Le QT, Tate D, Goffinet DR, et al. Dosimetric effects of patient displacement and collimator and gantry angle misalignment on intensity modulated radiation therapy. Radiother Oncol 2000 Jul;56(1):97-108.

13. Hanley J, Lumley MA, Mageras GS, Sun J, Zelefsky MJ, Leibel $\mathrm{SA}$, et al. Measurement of patient positioning errors in threedimensional conformal radiotherapy of the prostate. Int J Radiat Oncol Biol Phys 1997 Jan 15;37(2):435-44.

14. Fully three-dimensional image reconstruction from cone-beam sources. Proceedings of the conference on pattern recognition image processing. New York; 1978.

15. Nalcioglu O, Cho ZH. Reconstruction of 3-D objects from cone beam projections. Proc IEEE 1978;66(11):1584-5.

16. Jaffray DA, Siewerdsen JH, Wong JW, Martinez AA. Flat-panel cone-beam computed tomography for image-guided radiation therapy. Int J Radiat Oncol Biol Phys 2002 Aug 1;53(5):1337-49.

17. Groh BA, Siewerdsen JH, Drake DG, Wong JW, Jaffray DA. A performance comparison of flat-panel imager-based MV and $\mathrm{kV}$ cone-beam CT. Med Phys 2002 Jun;29(6):967-75.

18. Simpson RG, Chen CT, Grubbs EA, Swindell W. A 4-MV CT scanner for radiation therapy: The prototype system. Med Phys 1982 Aug;9(4):574-9.

19. Morton EJ, Swindell W, Lewis DG, Evans PM. A linear array, scintillation crystal-photodiode detector for megavoltage imaging. Med Phys 1991 Jul-Aug;18(4):681-91.

20. Ruchala KJ, Olivera GH, Schloesser EA, Mackie TR. Megavoltage CT on a tomotherapy system. Phys Med Biol 1999 Oct;44(10):2597-621. 
21. Mosleh Shirazi MA, Swindell W, Evans PM. Optimization of the scintillation detector in a combined 3D megavoltage CT scanner and portal imager. Med Phys 1998 Oct;25(10):1880-90.

22. Midgley S, Millar RM, Dudson J. A feasibility study for megavoltage cone beam CT using a commercial EPID. Phys Med Biol 1998 Jan;43(1):155-69.

23. Spies L, Ebert M, Groh BA, Hesse BM, Bortfeld T. Correction of scatter in megavoltage cone-beam CT. Phys Med Biol 2001 Mar;46(3):821-33.

24. Volumetric CT from an a-Si EPID. Sixth international workshop on portal imaging. Belgium; 2000.

25. Ford EC, Chang J, Mueller K, Sidhu K, Todor D, Mageras G, et al. Cone-beam CT with megavoltage beams and an amorphous silicon electronic portal imaging device: Potential for verification of radiotherapy of lung cancer. Med Phys 2002 Dec;29(12):291324.

26. Seppi EJ, Munro P, Johnsen SW, Shapiro EG, Tognina C, Jones $\mathrm{D}$, et al. Megavoltage cone-beam computed tomography using a high-efficiency image receptor. Int J Radiat Oncol Biol Phys 2003 Mar 1;55(3):793-803.

27. Anastasio MA, Shi D, Pan X, Pelizzari C, Munro P. A preliminary investigation of local tomography for megavoltage CT imaging. Med Phys 2003 Nov;30(11):2969-80.

28. Sidhu K, Ford EC, Spirou S, Yorke E, Chang J, Mueller K, et al. Optimization of conformal thoracic radiotherapy using conebeam CT imaging for treatment verification. Int J Radiat Oncol Biol Phys 2003 Mar 1;55(3):757-67.

29. Uematsu M, Shioda A, Suda A, Tahara K, Kojima T, Hama Y, et al. Intrafractional tumor position stability during computed tomography (CT)-guided frameless stereotactic radiation therapy for lung or liver cancers with a fusion of CT and linear accelerator (FOCAL) unit. Int J Radiat Oncol Biol Phys 2000 Sep $1 ; 48(2): 443-8$

30. Martinez AA, Yan D, Lockman D, Brabbins D, Kota K, Sharpe $\mathrm{M}$, et al. Improvement in dose escalation using the process of adaptive radiotherapy combined with three-dimensional conformal or intensity-modulated beams for prostate cancer. Int J Radiat Oncol Biol Phys 2001 Aug 1;50(5):1226-34.

31. Ruchala KJ, Olivera GH, Kapatoes JM, Schloesser EA, Reckwerdt PJ, Mackie TR. Megavoltage CT image reconstruction during tomotherapy treatments. Phys Med Biol 2000 Dec;45(12):354562.

32. Siewerdsen JH, Jaffray DA. Cone-beam computed tomography with a flat-panel imager: Magnitude and effects of X-ray scatter. Med Phys 2001 Feb;28(2):220-31.

33. Yu CX, Jaffray DA, Wong JW. The effects of intra-fraction organ motion on the delivery of dynamic intensity modulation. Phys Med Biol 1998 Jan;43(1):91-104.

34. Tome WA, Meeks SL, Buatti JM, Bova FJ, Friedman WA, Li Z. A high-precision system for conformal intracranial radiotherapy. Int J Radiat Oncol Biol Phys 2000 Jul 1;47(4):1137-43.

35. Tome WA, Meeks SL, McNutt TR, Buatti JM, Bova FJ, Friedman WA, et al. Optically guided intensity modulated radiotherapy. Radiother Oncol 2001 Oct;61(1):33-44.

36. Phillips MH, Singer K, Miller E, Stelzer K. Commissioning an image-guided localization system for radiotherapy. Int J Radiat Oncol Biol Phys 2000 Aug 1;48(1):267-76.

37. Bentel GC, Marks LB, Hendren K, Brizel DM. Comparison of two head and neck immobilization systems. Int J Radiat Oncol Biol Phys 1997 Jul 1;38(4):867-73.

38. Leavitt DD, Starkschall G, editors. The application of positional and dose distribution information in the treatment room. $12^{\text {th }}$ international conference on the use of computers in radiation therapy: Medical Physics Publishing; 1997.

39. Lattanzi J, McNeeley S, Pinover W, Horwitz E, Das I, Schultheiss TE, et al. A comparison of daily CT localization to a daily ultrasound-based system in prostate cancer. Int J Radiat Oncol Biol Phys 1999 Mar 1;43(4):719-25.

40. Mohan DS, Kupelian PA, Willoughby TR. Short-course intensitymodulated radiotherapy for localized prostate cancer with daily transabdominal ultrasound localization of the prostate gland. Int $\mathrm{J}$ Radiat Oncol Biol Phys 2000 Feb 1;46(3):575-80.

41. Hurkmans CW, Remeijer P, Lebesque JV, Mijnheer BJ. Set-up verification using portal imaging; Review of current clinical practice. Radiother Oncol 2001 Feb;58(2):105-20.

42. Huizenga H, Levendag PC, De Porre PM, Visser AG. Accuracy in radiation field alignment in head and neck cancer: A prospective study. Radiother Oncol 1988 Feb;11(2):181-7.

43. Mitine C, Dutreix A, van der Schueren E. Black and white in accuracy assessment of megavoltage images: The medical decision is often grey. Radiother Oncol 1993 Jul;28(1):31-6.

44. Willner J, Hadinger U, Neumann M, Schwab FJ, Bratengeier K, Flentje M. Three dimensional variability in patient positioning using bite block immobilization in 3D-conformal radiation treatment for ENT-tumors. Radiother Oncol 1997 Jun;43(3):31521.

45. Huddart RA, Nahum A, Neal A, McLean M, Dearnaley DP, Law $\mathrm{M}$, et al. Accuracy of pelvic radiotherapy: Prospective analysis of 90 patients in a randomised trial of blocked versus standard radiotherapy. Radiother Oncol 1996 Apr;39(1):19-29.

46. Stroom JC, Koper PC, Korevaar GA, van Os M, Janssen M, de 
Boer $\mathrm{HC}$, et al. Internal organ motion in prostate cancer patients treated in prone and supine treatment position. Radiother Oncol 1999 Jun;51(3):237-48

47. Bieri S, Miralbell R, Nouet P, Delorme H, Rouzaud M. Reproducibility of conformal radiation therapy in localized carcinoma of the prostate without rigid immobilization. Radiother Oncol 1996 Mar;38(3):223-30.

48. Van den Heuvel F, Verellen D. Correlating setup errors and patient individual parameters. Radiother Oncol 1996 Oct;41(1):95-6.

49. Bentel GC, Marks LB, Sherouse GW, Spencer DP, Anscher MS The effectiveness of immobilization during prostate irradiation. Int J Radiat Oncol Biol Phys 1995 Jan 1;31(1):143-8.

50. Soffen EM, Hanks GE, Hwang CC, Chu JC. Conformal static field therapy for low volume low grade prostate cancer with rigid immobilization. Int J Radiat Oncol Biol Phys 1991 Jan;20(1):1416.

51. Rosenthal SA, Galvin JM, Goldwein JW, Smith AR, Blitzer PH. Improved methods for determination of variability in patient positioning for radiation therapy using simulation and serial portal film measurements. Int J Radiat Oncol Biol Phys 1992;23(3):62-15.

52. van Herk M, Bruce A, Kroes AP, Shouman T, Touw A, Lebesque JV. Quantification of organ motion during conformal radiotherapy of the prostate by three dimensional image registration. Int $\mathrm{J}$ Radiat Oncol Biol Phys 1995 Dec 1;33(5):1311-20.

53. Mubata CD, Bidmead AM, Ellingham LM, Thompson V, Dearnaley DP. Portal imaging protocol for radical dose-escalated radiotherapy treatment of prostate cancer. Int J Radiat Oncol Biol Phys 1998 Jan 1;40(1):221-31.

54. Samson MJ, van Sornsen de Koste,J.R., de Boer HC, Tankink H, Verstraate M, Essers M, et al. An analysis of anatomic landmark mobility and setup deviations in radiotherapy for lung cancer. Int J Radiat Oncol Biol Phys 1999 Mar 1;43(4):827-32.

55. Balter JM, Ten Haken RK, Lawrence TS, Lam KL, Robertson JM. Uncertainties in CT-based radiation therapy treatment planning associated with patient breathing. Int J Radiat Oncol Biol Phys 1996 Aug 1;36(1):167-74.

56. Kubo HD, Hill BC. Respiration gated radiotherapy treatment: A technical study. Phys Med Biol 1996 Jan;41(1):83-91.

57. Bornstein BA, Cheng CW, Rhodes LM, Rashid H, Stomper PC, Siddon RL, et al. Can simulation measurements be used to predict the irradiated lung volume in the tangential fields in patients treated for breast cancer? Int J Radiat Oncol Biol Phys 1990 Jan;18(1):181-7.

58. Holmberg O, Huizenga H, Idzes MH, Lebesque JV, Vijlbrief RE, Mijnheer BJ. In vivo determination of the accuracy of field matching in breast cancer irradiation using an electronic portal imaging device. Radiother Oncol 1994 Nov;33(2):157-66.

59. Carter DL, Marks LB, Bentel GC. Impact of setup variability on incidental lung irradiation during tangential breast treatment. Int $\mathrm{J}$ Radiat Oncol Biol Phys 1997 Apr 1;38(1):109-15.

60. Creutzberg CL, Althof VG, Huizenga H, Visser AG, Levendag PC. Quality assurance using portal imaging: The accuracy of patient positioning in irradiation of breast cancer. Int $\mathrm{J}$ Radiat Oncol Biol Phys 1993 Feb 15;25(3):529-39.

61. De Neve W, Van den Heuvel F, Coghe M, Verellen D, De Beukeleer M, Roelstraete A, et al. Interactive use of on-line portal imaging in pelvic radiation. Int J Radiat Oncol Biol Phys 1993 Feb 15;25(3):517-24. 